\title{
Compulsão alimentar e a sua relação com o consumo alimentar durante o período da pandemia em mulheres acima de 50 anos
}

Binge eating and its relationship with food consumption during the pandemic period in women over 50 years old

Los atracones y su relación con el consumo de alimentos durante el período pandémico en mujeres mayores de 50 años

Recebido: 17/11/2021 | Revisado: 21/11/2021 | Aceito: 25/11/2021 | Publicado: 03/12/2021

\author{
Gabriella Rizzi Galleti \\ ORCID: https://orcid.org/0000-0002-4077-2318 \\ Centro Universitário de Brasília, Brasil \\ E-mail: gabriella.rizzi@sempreceub.com \\ Dayanne da Costa Maynard \\ ORCID: https://orcid.org/0000-0002-9295-3006 \\ Centro Universitário de Brasília, Brasil \\ E-mail: dayanne.maynard@ceub.edu.br
}

\begin{abstract}
Resumo
No início de 2020, uma pandemia chamada COVID-19, afetou a saúde do mundo, como também os hábitos alimentares, a frequência no consumo alimentar e a compulsão alimentar se fizeram presentes, dando aos indivíduos uma falsa sensação de prazer (com vista a diminuição dos danos). Nos momentos vividos em isolamento social, muitas mulheres se encontraram em desarmonia com um turbilhão de sentimentos novos, incluindo os efeitos da entrada no climatério. O presente estudo teve como objetivo analisar a compulsão alimentar e a sua relação com o consumo alimentar em mulheres a partir de 50 anos durante a pandemia. A pesquisa foi transversal e descritiva, avaliada por meio de questionários como o questionário de frequência alimentar e escala compulsão alimentar periódica, além de um questionário adaptado. A amostra foi composta por 50 participantes (mulheres em fase de climatério), onde foi constatado que apesar da alteração hormonal, a maioria dessas mulheres encontram-se em um estado de compulsão alimentar moderada, por serem capazes de controlar a quantidade/qualidade de alimentos e a periodicidade. Houve um aumento na frequência do consumo de todos os grupos alimentares Portanto, é possível concluir que a compulsão alimentar em mulheres acima de 50 anos durante a pandemia foi considerada moderada e que a mesma se dá por vários fatores, entre eles o estresse, dietas da moda sem orientação de um profissional especializado e condições emocionais. E que houve um aumento considerável na frequência e consumo em todos os grupos dos alimentos.
\end{abstract}

Palavras-chave: Compulsão alimentar; Hábitos alimentar; Frequência alimentar; Isolamento social; Mulheres.

\begin{abstract}
In early 2020, a pandemic called COVID-19 affected the health of the world, as well as eating habits, frequency of food consumption and binge eating were present, giving individuals a false sense of pleasure (with a view to decreasing damage). In moments lived in social isolation, many women found themselves in disharmony with a whirlwind of new feelings, including the effects of entering the climacteric. The present study aimed to analyze binge eating and its relationship with food consumption in women aged 50 years and over during the pandemic. The research was cross-sectional and descriptive, evaluated through questionnaires such as the food frequency questionnaire and binge eating scale, in addition to an adapted questionnaire. The sample consisted of 50 participants (menopause women), where it was found that despite the hormonal change, most of these women are in a state of moderate binge eating, as they are able to control the quantity/quality of food and periodicity. There was an increase in the frequency of consumption of all food groups. Therefore, it is possible to conclude that binge eating in women over 50 years old during the pandemic was considered moderate and that it is due to several factors, including stress, diets. fashion without the guidance of a specialized professional and emotional conditions. And that there has been a considerable increase in frequency and consumption in all food groups.
\end{abstract}

Keywords: Binge eating; Eating habits; Food frequency; Social isolation; Women.

\section{Resumen}

A principios de 2020, una pandemia llamada COVID-19 afectó la salud del mundo, además de los hábitos alimenticios, la frecuencia de consumo de alimentos y los atracones, dando a las personas una falsa sensación de placer (con miras a disminuir el daño). En momentos vividos en aislamiento social, muchas mujeres se encontraron en 
discordia con un torbellino de nuevos sentimientos, incluidos los efectos de entrar en el climaterio. El presente estudio tuvo como objetivo analizar los atracones y su relación con el consumo de alimentos en mujeres de 50 años y más durante la pandemia. La investigación fue transversal y descriptiva, evaluada a través de cuestionarios como el cuestionario de frecuencia alimentaria y la escala de atracones, además de un cuestionario adaptado. La muestra estuvo conformada por 50 participantes (mujeres menopáusicas), donde se encontró que a pesar del cambio hormonal, la mayoría de estas mujeres se encuentran en un estado de atracones moderados, ya que son capaces de controlar la cantidad / calidad de los alimentos y la periodicidad. Hubo un aumento en la frecuencia de consumo de todos los grupos de alimentos, por lo que es posible concluir que los atracones en mujeres mayores de 50 años durante la pandemia se consideraron moderados y que se deben a varios factores, entre ellos el estrés, la dieta. moda sin la guía de un profesional especializado y condiciones emocionales. Y que ha habido un aumento considerable en la frecuencia y el consumo en todos los grupos de alimentos.

Palabras clave: Atracones; Hábitos alimenticios; Frecuencia de alimentos; Aislamiento social; Mujeres.

\section{Introdução}

O ano de 2020 foi considerado anômalo, por causa do surgimento do coronavírus, SARS-Cov2, que reportou em pandemia. Seguindo orientações da Organização Mundial da Saúde (OMS, 2020) fez-se necessário o isolamento social que abrangeu a população mundial. Segundo Ghebreyesus (2020) os países foram chamados para ativar e intensificar mecanismos emergenciais de resposta, buscar casos suspeitos, isolar, testar e tratar todo episódio de COVID-19, além de traçar as pessoas que tiveram contato com ele.

Como consequência gerou uma síncope na saúde e economia de vários países. As pessoas estavam acostumadas a transitar com livre arbítrio, a uma rotina intensa de trabalho, lazer, estudos e outros. De maneira repentina se viram obrigadas a se isolar em casa para que o vírus não fosse transmitido a um número maior de habitantes. Com isso houve uma mudança drástica nos hábitos e comportamento das famílias, foi preciso reaprender o ato de conviver (Brito, 2020).

Antes do isolamento social era possível manter uma rotina alimentar mais facilmente, com horários de trabalho, tempos limitados para se alimentar, porém 2020 (dois mil e vinte) foi um ano atípico, em que as pessoas foram obrigadas a ficar em casa e com isso foi desencadeando o descontrole alimentar. Um meio de perceber que a alimentação está desequilibrada é identificar certos hábitos como, pular algumas refeições e exageros, entre outras, a fim de compensar a falta de alimento, esses descontroles alimentares podem se tornar gatilhos no desenvolvimento de compulsão alimentar (Silva, 2021).

A compulsão alimentar é um distúrbio caracterizado pela falta de controle. Existe uma vontade incontrolável, uma necessidade de comer, de ingerir alimentos, mesmo sem sentir fome. E mesmo estando satisfeita, a pessoa não para de comer, o que leva a ingerir enormes quantidades de alimentos em muito pouco tempo. Várias são as causas que levam a compulsão alimentar: estresse, dietas sem acompanhamento de um profissional especializado, mudanças hormonais e emocionais, isolamento social, dentre outros (Bloc, 2019). Nessas alterações os hormônios do estresse cortisol e adrenalina são liberados no sistema nervoso quando se apresenta algumas situações de desalento, desconforto como frustração, medo, irritação e tristeza causando desorganização na qualidade de vida, aliviar o estresse com alimentos industrializados remete a falsa sensação de bem-estar por focar em recompensa, pois o cérebro necessita do prazer imediato, com gordura e/ou açúcar. A opção de criar uma salada, descascar verduras, procurar ingredientes para temperar demanda tempo e disposição, adiando a gratificação instantânea (Campos, 2020).

Diante da sensibilidade a essas alterações ocasionadas pela menopausa, as mulheres se tornam mais susceptíveis a essas condições, essas que contribuem para que as mulheres tenham uma alimentação desequilibrada. Um fator que exemplifica esse comportamento seria a entrada da mulher no climatério (Curta, 2020). De acordo com a Sociedade Brasileira de Endocrinologia (2009), as mulheres nascem com dois ovários - e neles existem diversos folículos, de número limitado, que serão os futuros óvulos. Quando esse número termina ou está no final, o organismo entra na menopausa, fato que ocorre em torno dos 50 anos. Esses folículos produzem dois hormônios, o estrogênio e a progesterona. Por volta dos 40, 50 anos da 
mulher, a progesterona deixa de ser fabricada e o primeiro sintoma é a irregularidade menstrual (os ciclos ficam inicialmente mais curtos e depois ocorrem atrasos menstruais), além de irritabilidade, nervosismo e insônia. Essa fase inicial é chamada de climatério ou pré-menopausa.

Em tempos de isolamento social muitas mulheres enfrentam problemas, tais como a compulsão alimentar, isso por sua vez interfere na saúde física e mental, sendo necessário um acompanhamento com profissionais da saúde, como psicólogos, educadores físicos e nutricionistas. Nesta pandemia se intensificou mais a questão da pseudo ansiedade e se desenvolveu uma alimentação de maneira compulsiva. E para trabalhar esse tipo de transtorno, a presença do nutricionista é de suma importância. Diante do exposto esse presente estudo teve como objetivo analisar a compulsão alimentar e a sua relação com o consumo alimentar em mulheres a partir de 50 anos durante a pandemia.

\section{Metodologia}

Foi realizada uma pesquisa de campo do tipo observacional, transversal e descritiva. Nesta pesquisa foram recrutadas, adultos do sexo feminino, com idade de 50 anos, residentes em Brasília - DF, Natal - RN, Fortaleza - CE e Rio de Janeiro RJ. Foi estabelecido o seguinte critério de inclusão - mulheres na faixa etária a partir de 50 anos e que deram ciência ao termo de consentimento livre e esclarecido. Os critérios de exclusão foram mulheres analfabetas, questionários incompletos ou recusa em participação no estudo.

Procurando contribuir com o desenvolvimento do cenário da nutrição, esta pesquisa se encontra presente, perante os resultados obtidos pela aplicação do questionário e acompanhamento de mulheres na faixa etária de 50 anos ou mais, que se encontram na fase do climatério. Esta se divide em duas partes: a primeira parte ocorreu com a divulgação da pesquisa e seleção da amostra, já a segunda parte ocorreu pela aplicação de três questionários, sendo eles o questionário de frequência alimentar (QFA), a escala de compulsão alimentar periódica (ECAP) e um questionário sobre fatores e atividades na pandemia elaborado pela autora.

A pesquisa teve como amostra 50 mulheres escolhidas pela faixa etária, em cada região, a escolha por esses estados/cidades foi realizada de acordo com a disponibilidade de participação. Por meio de um questionário contendo 5 perguntas elaboradas antecipadamente tais como: questionamento sobre alimentação balanceada, como estava a alimentação da entrevistada antes da pandemia, o impacto que a pandemia causou na vida da mulher, refeições durante o distanciamento social, frequência no consumo de alimentos durante distanciamento social. O questionário criado para o estudo tem como objetivo analisar o comportamento alimentar em mulheres a partir de 50 anos durante a pandemia para verificar a relação da compulsão alimentar com o momento atual da COVID-19. As participantes tiveram acesso ao questionário através de e-mail e rede social, contendo na primeira página do formulário o Termo de Consentimento Livre e Esclarecido e as participantes somente conseguiam acessar as questões após consentirem com ele.

O questionário que foi aplicado (QFA) consiste em uma ferramenta que tem como propósito o parecer da dieta habitual de grupos populacionais e expõe como prós a velocidade na execução e eficiência na prática epidemiológica, para reconhecer o consumo habitual de alimentos além do baixo custo já o ECAP é um questionário autoaplicável, apropriado para distinguir pessoas conforme a gravidade da compulsão alimentar periódica. O mesmo possui três etapas, a primeira etapa seria para verificar as demonstrações de hábitos comportamentais, sentimentos e cognições, a segunda com afirmativas que refletiam a intensidade e valorados com pontuação, e a terceira etapa com afirmativas que correspondiam a frequência, a quantidade de alimento e o grau de emoção, com algumas afirmativas e valor de 0 a 3, após o somatório dos pontos é finalizado com a identificação do escore identificado com menor ou igual a 17= Sem CAP; entre 17 e 30= CAP Moderado; 30 ou mais= CAP Grave (Freitas et al., 2001). 
O cronograma para aplicação do questionário com o objetivo em análise ao comportamento alimentar em mulheres a partir de 50 anos durante a pandemia, questionário de frequência alimentar-QFA com ênfase nos alimentos consumidos antes e após a pandemia, como coleta de dados aconteceu na segunda quinzena do mês de junho/2021.

Para análise dos dados, foi utilizado o método da estatística descritiva e sua verificação foi feita por meio da frequência e cálculo do percentual, sendo demostrado por meio de tabelas.

A coleta de dados foi iniciada apenas após a aprovação do comitê de ética e Pesquisa do CEUB com o número 4.744.521 e ciência dos participantes do TCLE. Na execução e divulgação dos resultados foi garantido o total sigilo da identidade dos participantes e a não discriminação ou estigmatização dos sujeitos da pesquisa, além da conscientização dos sujeitos quanto à publicação de seus dados.

\section{Resultados e Discussão}

A seguir, as tabelas revelam os resultados obtidos através da aplicação dos questionários para análise do comportamento alimentar e compulsão alimentar em mulheres a partir de 50 anos durante a pandemia. Na tabela 1 que trata sobre os comportamentos alimentares das mulheres durante a pandemia, 50\% das voluntárias tinham, antes da pandemia, uma alimentação balanceada por conta própria, pois se baseavam em livros e revistas digitais, algumas receitas e refeições saudáveis com quantidades de calorias que entendiam ser ideal, isso sugere a preocupação delas com a qualidade de vida, ainda que seja suma importância a presença de um profissional capacitado. Observou-se que $42 \%$ das participantes, antes da pandemia, alternavam os dias durante a semana para se alimentar em casa, sinalizando que uma alimentação de melhor qualidade seria possível.

Percebe-se que $60 \%$ das mulheres questionadas sofreram um alto impacto na alimentação com a pandemia, pois começaram a reaprender o gosto pela gastronomia e cozinhar as próprias refeições. Sendo assim, $62 \%$ das mulheres preparavam suas próprias refeições, indicando primar pela qualidade e o gosto pessoal criando suas próprias combinações. Notou-se que a frequência no consumo de alimentos durante o distanciamento social, 56\% se alimentavam com um menor intervalo de tempo, sendo a maioria das mulheres questionadas, o que serve de alerta para alterações de peso e doenças (Tabela $1)$.

Segundo Souza (2021), foi realizada uma pesquisa a respeito de hábitos alimentares antes e durante a pandemia, no qual foi observado que durante o isolamento social houve uma diminuição nas refeições diurnas seguindo compensadas pelas refeições noturnas. Em contrapartida de acordo com as 50 mulheres respondentes à esta pesquisa, observou-se uma diminuição no intervalo de tempo entre as refeições, salientando que o aumento no consumo dos alimentos ocorreu independente dos turnos mencionados, e compactuando com a relação de possível aumento de peso.

De acordo com Santos (2020), em sua pesquisa sobre comportamento alimentar na pandemia, foi constatado um consumo considerável elevado de refeições prontas industrializadas, e uma diminuição nos produtos in natura. Percebe-se que por consequência a faixa etária dos respondentes ser entre 20 a 29 anos, esse consumo se fez constante junto ao Delivery. O que segue oposto a pesquisa aqui em questão, onde a população foram mulheres maduras acima de 50 anos preocupadas com alimentação preparada em casa, por elas mesmas e com uma regularidade frequente. 
Tabela 1. Dados dos comportamentos alimentares em mulheres a partir de 50 anos durante a pandemia sobre o nível de impacto/ Preparo das refeições/ Alimentação balanceada/ Frequência. Brasília-DF, 2021.

\begin{tabular}{|c|c|c|c|}
\hline & & $\mathbf{N}$ & $\%$ \\
\hline $\begin{array}{l}\text { Você tinha uma } \\
\text { alimentação } \\
\text { balanceada antes da }\end{array}$ & $\begin{array}{l}\text { sim (com orientação de } \\
\text { um profissional da área } \\
\text { de nutrição) }\end{array}$ & 15 & $30 \%$ \\
\hline pandemia? & sim (por conta própria) & 25 & $50 \%$ \\
\hline & as vezes & 8 & $16 \%$ \\
\hline & não & 2 & $4 \%$ \\
\hline & Não conheço nenhuma. & 17 & $10 \%$ \\
\hline Como se dava a sua & Em casa (todos os dias) & 15 & $30 \%$ \\
\hline $\begin{array}{l}\text { alimentação antes da } \\
\text { pandemia }\end{array}$ & $\begin{array}{l}\text { Em casa (algumas vezes } \\
\text { na semana) }\end{array}$ & 21 & $42 \%$ \\
\hline & $\begin{array}{l}\text { Em casa (algumas vezes } \\
\text { no mês) }\end{array}$ & 11 & $22 \%$ \\
\hline & Em casa (nunca) & 3 & $6 \%$ \\
\hline Qual foi o nível de & Alto & 30 & $60 \%$ \\
\hline impacto que a & Moderado & 11 & $22 \%$ \\
\hline pandemia teve sobre a & Baixo & 9 & $18 \%$ \\
\hline sua alimentação? & Indiferente & 0 & $0,0 \%$ \\
\hline $\begin{array}{l}\text { Assinale a alternativa } \\
\text { que retrata a sua }\end{array}$ & $\begin{array}{l}\text { Preparo minhas refeições } \\
\text { em casa }\end{array}$ & 31 & $62 \%$ \\
\hline $\begin{array}{l}\text { produção de suas } \\
\text { refeições durante o } \\
\text { distanciamento social: }\end{array}$ & $\begin{array}{l}\text { Outro integrante da } \\
\text { minha casa que prepara } \\
\text { as refeições }\end{array}$ & 8 & $16 \%$ \\
\hline & $\begin{array}{l}\text { Faço minhas refeições } \\
\text { em restaurantes }\end{array}$ & 6 & $12 \%$ \\
\hline & $\begin{array}{l}\text { Compro marmitas } \\
\text { prontas }\end{array}$ & 5 & $10 \%$ \\
\hline $\begin{array}{l}\text { Qual é a frequência no } \\
\text { consumo de alimentos }\end{array}$ & $\begin{array}{l}\text { Me alimento com mais } \\
\text { intervalo de tempo }\end{array}$ & 9 & $18 \%$ \\
\hline $\begin{array}{l}\text { durante o } \\
\text { distanciamento social? }\end{array}$ & $\begin{array}{l}\text { Me alimento com menos } \\
\text { intervalo de tempo }\end{array}$ & 28 & $56 \%$ \\
\hline & $\begin{array}{l}\text { Me alimento da mesma } \\
\text { forma e quantidade antes } \\
\text { do distanciamento social }\end{array}$ & 8 & $16 \%$ \\
\hline & $\begin{array}{l}\text { Não mensuro a } \\
\text { frequência que me alime }\end{array}$ & 5 & $10 \%$ \\
\hline
\end{tabular}

Fonte: Autores.

No trabalho realizado por Maynard et al. (2020), que analisou o padrão alimentar durante o distanciamento social e a relação com a ansiedade, encontrou que após analisar 1000 indivíduos o consumo alimentar de indivíduos ansiosos foi mais prevalente para alimentos considerados não saudáveis. Dados esses muito importantes para alertar a população em relação as mudanças alimentares impostas durante a pandemia, e os cuidados que devem ser tomados após o retorno das atividades, minimizando os prejuízos e a probabilidade do aumento de doenças crônicas não-transmissíveis.

Assim como no questionário anterior, a amostra se deu também com as mesmas 50 mulheres a partir de 50 anos, para obter parâmetros do consumo alimentar antes e durante o início da pandemia. Os alimentos foram distribuídos por categorias/grupos alimentares, tendo como base o Guia Alimentar da População Brasileira - Massa/raízes/cereais, Hortaliças/verduras/legumes, Frutas, Leite e derivados, Leguminosas, Óleos e gorduras, Carne e ovos, Açúcares e doces e diversos. A análise das tabelas, levou em consideração a maior frequência diária/porcentagem referentes aos resultados e discussões dos questionários de frequência alimentar - QFA antes e após o início da pandemia. 
Foi observado entre as participantes que antes da pandemia no grupo de Massa/ raízes/cereais, 36\% das participantes consumiam macarrão e batata doce 2 vezes ou mais por dia (Tabela 2). Porém, durante a pandemia foi registrado o consumo de macarrão em $52 \%$ das respondentes (Tabela 3). Isso representa um aumento de $16 \%$. É necessário atenção pois, apesar dos carboidratos serem fontes de energia para o nosso organismo, estes, elevam a glicose logo após o consumo sendo necessário moderação (Carvalho, 2008).

Já na categoria de Hortaliças/Verduras/Legumes, 36\% das mulheres ingeriam abóbora 2 vezes ou mais por dia antes da pandemia (Tabela 2). Durante a pandemia foi percebido o consumo da abóbora em 50\% (Tabela 3). A abóbora é um legume com poucas calorias, rico em fibras e ótimo aliado no auxílio de perda de peso, promove a sensação de saciedade por um espaço maior de tempo (Bernaud, 2013).

Em relação ao grupo de Frutas, os destaques de consumo de banana foram de 36\% antes da pandemia 2 ou mais vezes por dia (Tabela 2). Já durante a pandemia 44\% das mulheres consumiram banana na mesma frequência (Tabela 3), com aumento de $8 \%$. Essa fruta auxilia na prevenção de algumas doenças cardiovasculares, proteção ao sistema digestivo e redução de câimbras, mas como ocorre com outros alimentos, pode contribuir com ganho de peso caso seja consumido em excesso (Santos \& Scalon, 2020).

No grupo de Leite e derivados, $68 \%$ consumiam queijo uma vez ao dia antes da pandemia (Tabela 2) e durante a pandemia $80 \%$ fazia uso de queijo uma vez ao dia (Tabela 3). Vale ressaltar que o queijo contém vitaminas, minerais além de proteínas com benefícios à saúde, mas se faz necessário o cuidado com excesso na ingestão já que há presença de gorduras saturadas e sódio que podem causar danos à saúde (Siqueira, 2013).

Já em relação ao grupo de óleos e gorduras, antes da pandemia, houve consumo de 50\% de maionese uma vez por semana (Tabela 2). Contudo, durante a pandemia 62\%, também uma vez por semana, faziam uso de maionese (Tabela 3). Quando consumida com moderação não faz mal pois, é fonte de nutrientes como gorduras saudáveis e vitaminas A, D, E e K (Furlanetto, 1982).

No grupo de Carnes, o destaque foi para os embutidos (presunto, mortadela, salsicha, salame e linguiça) ao qual $46 \%$ das participantes consumiam esses alimentos uma vez ao dia, antes da pandemia (Tabela 2). Foi observado, que durante a pandemia houve um registro de 54\% no consumo de embutidos uma vez ao dia (Tabela 3), o que resulta numa diferença de 8\%. O que serve de cautela, porque de acordo com a Organização Mundial da Saúde (OMS) - 2015 os embutidos aumentam a chance de desenvolvimento de câncer (Louzada, 2015).

No grupo de Açúcares e doces, $66 \%$ consumiram doces (goiabada, bananada, marmelada etc.) duas ou mais vezes ao dia, antes da pandemia (Tabela 2). Já durante a pandemia, foi registrado que $82 \%$ faziam uso duas ou mais vezes ao dia (Tabela 3). Com aumento de 16\%, um índice preocupante, visto que o consumo frequente e desmedido é prejudicial à saúde (Slater, 2006).

No grupo de Diversos o mais consumido foi o refrigerante com $66 \%$ antes da pandemia, em uma frequência de duas ou mais vezes no dia (Tabela 2). E como verificado o registro durante a pandemia foi de $86 \%$ (Tabela 3). Como mencionado acima é recomendável a não utilização do produto, por conter componentes que também faz mal ao organismo e valor nutricional nulo (Slater, 2006). 
Tabela 2. Análise do consumo alimentar as participantes antes da Pandemia de COVID-19. Brasília-DF, 2021.

\begin{tabular}{|c|c|c|c|c|}
\hline & & & $\mathrm{N}$ & $\%$ \\
\hline Massas/raízes & Arroz & $1 \mathrm{vez}$ por semana & 13 & $26 \%$ \\
\hline \multirow[t]{2}{*}{ /cereais } & Macarrão & 2 vezes ou mais por dia & 18 & $36 \%$ \\
\hline & Batata Doce & 2 vezes ou mais por dia & 18 & $36 \%$ \\
\hline \multirow{3}{*}{$\begin{array}{l}\text { Hortaliças/verduras/l } \\
\text { egumes }\end{array}$} & Alface & 1 vez por dia & 13 & $26 \%$ \\
\hline & Agrião/Rúcula & 1 vez por dia & 16 & $32 \%$ \\
\hline & Abóbora & 2 vezes ou mais por dia & 18 & $36 \%$ \\
\hline \multirow[t]{3}{*}{ Frutas } & Abacate & 1 a 3 vezes por mês & 15 & $30 \%$ \\
\hline & Banana & 2 vezes ou mais por dia & 18 & $36 \%$ \\
\hline & Mamão & 1 vez por dia & 18 & $36 \%$ \\
\hline \multirow[t]{3}{*}{ Leite e derivados } & Leite integral & 1 vez por dia & 23 & $46 \%$ \\
\hline & Iogurte & 1 vez por dia & 26 & $52 \%$ \\
\hline & Queijo & 1 vez por dia & 34 & $68 \%$ \\
\hline \multirow[t]{3}{*}{ Óleos e gorduras } & Maionese & 1 vez por semana & 25 & $50 \%$ \\
\hline & Manteiga/margarina & 1 vez por dia & 17 & $34 \%$ \\
\hline & Óleo & 2 vezes ou mais por dia & 20 & $40 \%$ \\
\hline \multirow[t]{3}{*}{ Carne } & Carne Bovina & 2 a 4 vezes por semana & 15 & $30 \%$ \\
\hline & Frango & $1 \mathrm{vez}$ por semana & 14 & $28 \%$ \\
\hline & $\begin{array}{l}\text { Alimento: } \quad \begin{array}{l}\text { Embutidos } \\
\text { (presunto, }\end{array} \\
\text { salsicha, salame, linguiça) }\end{array}$ & 1 vez por dia & 23 & $46 \%$ \\
\hline \multirow[t]{3}{*}{ Açúcares e doces } & Açúcar & 2 vezes ou mais por dia & 32 & $64 \%$ \\
\hline & $\begin{array}{l}\text { Doces (goiabada, bananada, } \\
\text { marmelada, etc.) }\end{array}$ & 2 vezes ou mais por dia & 33 & $66 \%$ \\
\hline & Chocolate/Brigadeiro & 2 vezes ou mais por dia & 31 & $62 \%$ \\
\hline \multirow[t]{3}{*}{ Diversos } & Café & 2 vezes ou mais por dia & 32 & $64 \%$ \\
\hline & Refrigerante & 2 vezes ou mais por dia & 33 & $66 \%$ \\
\hline & Suco Natural & 2 vezes ou mais por dia & 22 & $44 \%$ \\
\hline
\end{tabular}

Fonte: Autores (2021).

Conforme afirma Silva, Prado e Seixas (2016), apesar da diferença no conceito de hábito e comportamento, estes se concatenam. No estudo em questão, o comportamento alimentar é relacionado a fatores emocionais o que é compensado pela mudança de hábito na ingestão dos alimentos, quanto à qualidade, quantidade e frequência.

Como aponta Mazzolani (2021), pelo fato das mulheres estarem submetidas ao isolamento social em decorrência da pandemia, houve uma alteração nos hábitos alimentares de todas as participantes principalmente quanto à frequência, afirma ainda que essa mudança se deu em mulheres obesas ou eutróficas, apesar que a escolha alimentar já ocorria, cada uma com motivos distintos, antes da pandemia. A autora salienta que o "comer emocional" aparece de forma mais acentuada nas respondentes obesas. Em contrapartida na análise do que foi verificado no resultado das tabelas em questão, a alteração no comportamento alimentar e/ou mesmo no "comer emocional" aconteceu nas participantes como um todo, independente da estética e peso ideal. 
Tabela 3. Análise do consumo alimentar as participantes durante a Pandemia de COVID-19. Brasília-DF, 2021.

\begin{tabular}{|c|c|c|c|c|}
\hline & & & $\mathrm{N}$ & $\%$ \\
\hline \multirow{3}{*}{$\begin{array}{l}\text { Massas/raízes } \\
\text { /cereais }\end{array}$} & Arroz & 2 vezes ou mais por dia & 20 & $40 \%$ \\
\hline & Macarrão & 2 vezes ou mais por dia & 26 & $52 \%$ \\
\hline & Batata Doce & 2 vezes ou mais por dia & 19 & $38 \%$ \\
\hline \multirow{3}{*}{$\begin{array}{l}\text { Hortaliças/verduras } \\
\text { /legumes }\end{array}$} & Alface & 2 vezes ou mais por dia & 26 & $52 \%$ \\
\hline & Agrião/Rúcula & 1 vez por dia & 17 & $34 \%$ \\
\hline & Abóbora & 1 vez por dia & 25 & $50 \%$ \\
\hline \multirow[t]{3}{*}{ Frutas } & Abacate & 1 vez por dia & 15 & $30 \%$ \\
\hline & Banana & 2 vezes ou mais por dia & 22 & $44 \%$ \\
\hline & Mamão & 2 vezes ou mais por dia & 24 & $48 \%$ \\
\hline \multirow[t]{3}{*}{ Leite e derivados } & Leite integral & 1 vez por dia & 17 & $34 \%$ \\
\hline & Iogurte & 2 vezes ou mais por dia & 36 & $72 \%$ \\
\hline & Queijo & 1 vez por dia & 40 & $80 \%$ \\
\hline \multirow[t]{3}{*}{ Óleos e gorduras } & Maionese & $1 \mathrm{vez}$ por semana & 32 & $64 \%$ \\
\hline & Manteiga/margarina & 2 vezes ou mais por dia & 38 & $76 \%$ \\
\hline & Óleo & 2 vezes ou mais por dia & 32 & $64 \%$ \\
\hline \multirow[t]{3}{*}{ Carne } & Carne Bovina & 2 vezes ou mais por dia & 18 & $36 \%$ \\
\hline & Frango & 2 vezes ou mais por dia & 14 & $28 \%$ \\
\hline & $\begin{array}{l}\text { Alimento: } \quad \begin{array}{l}\text { Embutidos } \\
\text { (presunto, }\end{array} \text { mortadela, } \\
\text { salsicha, salame, linguiça) }\end{array}$ & 2 vezes ou mais por dia & 27 & $54 \%$ \\
\hline \multirow[t]{3}{*}{ Açúcares e doces } & Açúcar & 2 vezes ou mais por dia & 41 & $82 \%$ \\
\hline & $\begin{array}{l}\text { Doces (goiabada, bananada, } \\
\text { marmelada, etc.) }\end{array}$ & 2 vezes ou mais por dia & 40 & $80 \%$ \\
\hline & Chocolate/Brigadeiro & 2 vezes ou mais por dia & 35 & $70 \%$ \\
\hline \multirow[t]{3}{*}{ Diversos } & Café & 2 vezes ou mais por dia & 34 & $68 \%$ \\
\hline & Refrigerante & 2 vezes ou mais por dia & 43 & $86 \%$ \\
\hline & Suco Natural & 2 vezes ou mais por dia & 25 & $50 \%$ \\
\hline
\end{tabular}

Fonte: Autores (2021).

Na Tabela 4 apresenta-se ECAP-Escala Compulsão Alimentar Periódica ainda com a amostra das 50 mulheres a partir de 50 anos. Os resultados mostram que 46\% das mulheres avaliadas possuíam Compulsão alimentar periódica moderada. Já, $16 \%$ possuem CAP grave, que não conseguem controlar o que comem e nem mesmo a quantidade, comem até se sentirem desconfortáveis de tão cheios, engolem a comida sem mastigar e rápido, comem sozinho, escondido pela vergonha da quantidade de alimento seguida da sensação de culpa e com inúmeros episódios por semana.

Tabela 4. Distribuição da classificação da compulsão alimentar das participantes. Brasília-DF, 2021.

\begin{tabular}{llcc}
\hline & & $\mathrm{N}$ & $\%$ \\
\hline Escala de compulsão & Sem CAP & 19 & $38 \%$ \\
& CAP moderada & 23 & $46 \%$ \\
& CAP grave & 8 & $16 \%$ \\
\hline
\end{tabular}


Segundo Freitas et al. (2001), a ECAP é um instrumento aplicado com o propósito de avaliar o nível da compulsão periódica, de acordo com hábitos e comportamento alimentar, servindo como diagnose para auxílio ao tratamento caso seja mensurado.

Constatou-se que alguns hábitos e comportamentos alimentares ficaram evidentes após o preenchimento do ECAP, uma porção das mulheres têm o hábito de engolir a comida sem realmente mastigá-la. Quando isso acontece, em geral se sentem desconfortáveis, empanturradas com a sensação de ter comido demais, o importante é observar com atenção os sintomas, pois em alguns casos podem desenvolver desconfortos na região do estômago e abdômen. Observou-se também que se sentem incapazes de controlar o comportamento alimentar, entram em desespero tentando manter o controle, esse é o sentimento de muitas participantes, é necessário levar em consideração o estado emocional, identificar o gatilho e traçar estratégias na tentativa de controlar essa conduta (Azevedo, 2004).

Constatou-se que as participantes de vez em quando comem alguma coisa por impulso, mesmo não estando com fome, o ócio, o tédio e a compensação nas emoções podem contribuir para ingestão involuntária de alimentos. As atividades físicas podem servir de auxílio para que esse comportamento não se evidencie. Notou-se ainda que cerca de uma vez por mês, elas comem uma tal quantidade de comida e acabam se sentindo empanturradas, vários são os fatores que contribuem para a sensação de empachamento como, comer rápido, engolir sem mastigar direito, ingestão de alimentos que sobrepesam o sistema digestivo ou até mesmo a quantidade comida. É considerável a busca por um profissional da área para uma reeducação alimentar, caso se faça necessário. Percebeu-se que, depois de comer demais, tentam fazer uma redução na ingesta calórica, para compensar o excesso de calorias ingeridas, a causa dessa compensação pode estar atrelada a um sentimento de culpa e frustração por consumir calorias em excesso. Com o objetivo de atenuar o sentimento de ressarcimento e dano, existem algumas sugestões para retomar uma alimentação adequada como, eliminar as toxinas, realizar exercícios físicos, entre outras, sempre com orientação de um profissional da área (Aro, 2021).

Além disso, constatou-se que quando estão com outras pessoas, sentem-se constrangidas e não comem a mesma quantidade que o habitual, com isso fica evidente a consciência que têm em saber que é preciso haver um controle comportamental, mesmo que por vezes se sentem impotentes, o que remete novamente à busca por profissionais para auxiliar. Sendo assim, as mulheres, relatam que mesmo que pudessem saber quantas calorias deveriam ingerir, não têm ideia de qual seria a quantidade "normal" por dia ou por refeição. Como em várias áreas da vida tudo é uma questão de equilíbrio, e na alimentação não é diferente, existem motivos distintos perca de peso, ganho de peso, doenças, a quantidade de calorias depende muito de cada pessoa e do objetivo em vigor, o essencial é ter um acompanhamento de um profissional para um planejamento alimentar adequado (Viana, 2014).

Assim como a constatação de Garcês (2021), o estudo em questão reforça que o isolamento social em consequência da pandemia COVID-19, contribuiu de maneira negativa com o predomínio da compulsão alimentar, com o aumento do 'comer em resposta ao estresse', 'comer quando entediado' enfim o 'comer emocional', resultante do cenário atual, das incertezas e medos que trazem efeitos corporais como vazio no estômago, pressão no peito entre outras sensações. Houve um aditamento nos episódios de compulsão alimentar, pois algumas das respondentes já sinalizavam um desalinho na alimentação, anterior ao isolamento social. O público de Garcês refere-se a adultos obesos, em contrapartida o nicho do estudo aqui apresentado é dirigido às mulheres acima 50 anos.

De acordo com Aro (2021), em sua pesquisa também a respeito do comportamento alimentar durante pandemia por Covid-19, foi constatado que em decorrência da pandemia observou-se que mais da metade dos participantes comeram em maior quantidade por fatores emocionais, aumentando o hábito de degustar em menor espaço de tempo, com preferência para os doces e alimentos ultra processados, com isso observou-se um ganho de peso corporal, como sugerido presente pesquisa. 


\section{Conclusão}

Por meio dessa pesquisa conclui-se que a mudança no comportamento alimentar em mulheres acima de 50 anos se dá por vários fatores. Isso se intensificou durante o isolamento social em consequência da pandemia do COVID-19, com um efeito nocivo, pois o tempo excessivo em casa, a preocupação com um futuro incerto, desencadeou ainda mais os agentes que levam ao transtorno alimentar.

De acordo com o resultado da ECAP - Escala de Compulsão Alimentar Periódica, constatou-se que a maioria das mulheres dispõem de CAP moderada e apresentam episódios regulares de compulsão alimentar por semana, sendo relevante o controle da frequência dos episódios compulsivos e por consequência o controle do peso. Foi possível evidenciar no questionário de frequência alimentar - QFA que houve um aumento considerável na frequência e consumo no grupo dos alimentos Massa/ raízes/cereais, Hortaliças/Verduras/Legumes, Frutas, Leite e derivados, Óleos e gorduras, Carnes, Açúcares e doces e Diversos, constata-se que os fatores emocionais, o ócio e até mesmo dietas descontroladas influenciaram em um consumo desmedido, podendo ocasionar malefícios a saúde.

Algumas sugestões para a melhoria dessa condição comportamental, seriam exercícios físicos, palestras/cursos online, escutar música, ler um livro, administrar melhor o tempo, planejamento da frequência e escolha dos alimentos a serem preparados, se hidratar bem pois a região do cérebro que comanda a fome é a mesma que controla a sede, para que não haja confusão entre ambos. Assim também, devido a importância do tema, se faz necessário mais estudos para que com o conhecimento adquirido seja possível ajudar a população a minimizar os prejuízos causados diante da pandemia de Covid-19.

\section{Agradecimentos}

A equipe agradece a todas as participantes que responderam aos questionários.

\section{Referências}

Aro, F., Pereira, B. V., \& Bernardo, D. N. A. (2021). Comportamento alimentar em tempos de pandemia por Covid-19. Brazilian Journal of Development, 7 (6), https://DOI:10.34117/bjdv7n6-386.

Azevedo, A. P., Santos, C. C., \& Fonseca, D. C. (2004). Transtorno da compulsão alimentar periódica Archives of Clinical Psychiatry,31 (4), https://doi.org/10.1590/S0101-60832004000400008.

Bernaud, F. S. R., \& Rodrigues, T. C. (2013). Fibra alimentar: Ingestão adequada e efeitos sobre a saúde do metabolismo. Arquivos Brasileiros de Endocrinologia \& Metabologia, 57 (6), https://doi.org/10.1590/S0004-27302013000600001.

Bloc, L. G., Nazareth, A. C. P., Melo, A. K. S., \& Moreira, V. (2019). Transtorno de compulsão alimentar: revisão sistemática da literatura. Revista Psicologia e Saúde, 11 (1), http://dx.doi.org/10.20435/pssa.v11i1.617,

Brito, S. B. P., Braga, I.O., Cunha, C. C., \& Palácio, M. A. V. (2020). Takenami, Iukary. Pandemia da COVID-19: o maior desafio do século XXI Revista Visa em Debate Sociedade, ciência e tecnologia, 8 (2),

https://doi.org/10.22239/2317-269X.01531.

Campos, L. S., Leonel, C. F. S., \& Gutierrez, D. M. D. (2020). Relação entre estresse e obesidade: Uma revisão narrativa. BIUS-Boletim Informativo Unimotrisaúde em Sociogerontologia, 22 (16), BIUS/novembro/2020.

Carvalho, G. Q., \& Alfenas, R. C. G. (2008). Índice glicêmico: uma abordagem crítica acerca de sua utilização na prevenção e no tratamento de fatores de risco cardiovasculares. Brazilian Journal of Nutrition, Revista de Nutrição, 21 (5) https://doi.org/10.1590/S1415-52732008000500010.

Curta, J. C., \& Weissheimer, A. M. (2020). Percepções e sentimentos sobre as alterações corporais de mulheres climatéricas. Revista Gaúcha de Enfermagem, 41 (esp), https://doi.org/10.1590/1983-1447.2020.20190198.

Freitas, S., Lopes, C. S., Coutinho, W., \& Appolonario, J. C. (2001). Tradução e adaptação para o português da Escala de Compulsão Alimentar Periódica. Brazilian Journal of Psychiatry, 23 (4), https://doi.org/10.1590/S1516-44462001000400008.

Furlanetto, S. M. P., Lacerda, A. A., \& Campos, M. L. C. (1982). Pesquisa de alguns microrganismos em saladas com maionese adquiridas em restaurantes, lanchonetes e "rotisseries". Revista de Saúde Pública, 16 (6), https://doi.org/10.1590/S0034-89101982000600001.

Garcês, C. P., Efeitos negativos do período de isolamento social causado pela pandemia de COVID-19 no comportamento sedentário, nível de atividade física e compulsão alimentar em adultos com sobrepeso e obesidade, (Trabalho de Conclusão de Curso da Faculdade de Educação Física e Fisioterapia), Universidade Federal de Uberlândia, 2021. 
Ghebreyesus, T. A. (2020). Atendendo às necessidades de saúde mental: uma parte integrante da resposta COVID -19. World Psychiatry, 19 (2), 129-130, https://doi.org/10.1002/wps.20768.

Louzada, M. L. C., Martins, A. P. B., Baraldi, L. G., Levy, R. B., Claro, R. M., Moubarac, J. C., Cannon, G., \& Monteiro, C. A. (2015). Alimentos ultraprocessados e perfil nutricional da dieta no Brasil. Revista de Saúde Pública, 49, https://doi.org/10.1590/S0034-8910.2015049006132.

Mazzolani, B. C., Smaira, F. I., Esteves, G. P., André, H. C. S., Amarante, M. C., Castanho, D., Campos, K., Benatti, F. B., Pinto, A. J., Rodchel, H., Gualano, B. \& Fereeira, C. N. (2021). Influência do estado nutricional nos hábitos alimentares e determinantes da escolha alimentar entre mulheres brasileiras durante a pandemia de COVID-19. Frontiers in Nutrition, Brief research report, 8 (672372), https://doi.org/10.3389/fnut.2021.672372

Maynard, D. C., Anjos, H. A., Magalhães, A. C. V., Grimes, L. N., Costa, M. G. O., \& Santos, R. B. (2020). Consumo alimentar e ansiedade da população adulta durante a pandemia do COVID-19 no Brasil. Research, Society and Development, 9 (11), 1-22, https://doi.org/10.33448/rsd-v9i11.9905.

Santos, C. C., \& Scalon, S. P. Q. (2020). Efisiologia e Nutrição de Espécies Frutíferas e Arbóreas. Pantanal editora 1 (10), https://doi.org/10.46420/9786588319314.

Santos, R. S. M., \& Ayres, E. M. M. (2020). Impacto da Pandemia de Coronavírus (COVID-19) no Comportamento Alimentar. SEMEAR: Revista da Alimentação, Nutrição e Saúde, 2(3), 24-27, Universidade Federal do Estado do Rio de Janeiro-Escola de Nutrição.

Silva, J. K., Prado, S. D., \& Seixas, C. M. (2016). Comportamento alimentar no campo da Alimentação e Nutrição: do que estamos falando? Physis: Revista de Saúde Coletiva, 26 (4), https://doi.org/10.1590/S0103-73312016000400003.

Silva, T. S. S., Lima, E.R., Vilela, A. B. A., Rodrigues, V. P., \& Boery, R. N. S. O. (2021). Implicações da pandemia de COVID-19 nos hábitos alimentares de brasileiros: revisão integrativa Research, Society and Development, 10 (4), https://doi.org/10.33448/rsd-v10i4.14125.

Siqueira, A. M. O., Machado, E. C. L., \& Stamford. T. L. M. (2013). Bebidas lácteas com soro de queijo e frutas. Ciência Rural, 43 (9), https://doi.org/10.1590/S0103-84782013000900025.

Souza, T., Oliveira, L., Daniel, M., Ferreira, L., Della Lucia, C., Liboredo, J., \& Anastácio, L. (2021). Estilo de vida e hábitos alimentares antes e durante a quarentena de COVID-19 no Brasil. Nutrição em saúde pública, 1-(11). https://doi.org/10.1017/S136898002100255X.

Slater, B., Silva, M. V., Toral, N., \& Carmo, B. C. (2006). Consumo de doces, refrigerantes e bebidas com adição de açúcar entre adolescentes da rede pública de ensino de Piracicaba, São Paulo. Revista Brasileira de Epidemiologia, 9 (1), 121-30, https://www.scielosp.org/pdf/rbepid/2006.v9n1/121-130/pt.

Viana, D. S. O., Análise nutricional das dietas da moda, (TCC-Trabalho de Conclusão de Curso da Faculdade de Ciências da educação e Saúde), Centro Universitário de Brasília-Uniceub, 2014. 